\title{
Les élections démocratiques au Niger
}

\author{
Par Aichatou Dodo
}

\section{La souveraineté du peuple et les partis politiques}

Aux termes de l'article 5 de la Constitution nigérienne, "la souveraineté appartient au peuple... Aucune fraction du peuple ni aucun individu ne peut s'en attribuer l'exercice". L'article 7 ajoute que "le peuple exerce sa souveraineté par ses représentants élus et par voie de référendum". Selon les dispositions de ces deux articles, le peuple est l'arbitre dans la compétition permanente pour la conquête du pouvoir entre les partis politiques. Cette souveraineté du peuple sera étudiée autour de trois points: le suffrage, le régime des élections et les partis politiques.

\section{A. Le suffrage}

Il faut distinguer la jouissance du droit de vote qui donne au citoyen la qualité d'électeur et l'exercice du droit de vote qui ne s'acquiert qu'à la condition d'être inscrit sur une liste électorale.

\section{La jouissance du droit de vote}

L'article 8 de la Constitution nigérienne indique que: "... Sont électeurs, dans les conditions déterminées par la loi, les Nigériens des deux sexes, âgés de dix-huit (18) ans accomplis au jour du scrutin, ou mineurs émancipés jouissant de leurs droits civiques et politiques". L'article 6 du code électoral ajoute ${ }^{1}$ que les Nigériens ainsi désignés, ne doivent pas être dans l'un des cas d'incapacité prévus par la loi. Ces deux textes posent, en résumé, trois conditions cumulatives pour que le citoyen jouisse de son droit de vote.

\section{a) La condition de nationalité}

Contrairement à l'article 38 alinéa 2 de la Constitution qui impose que tout candidat à la présidence de la République du Niger soit Nigérien d'origine ${ }^{2}$, les articles 8 de la Consti-

1 Il reprend intégralement les dispositions de l'article 8 de la Constitution.

2

La nationalité d'origine a fait "couleur beaucoup d'encre", pourtant le code de la nationalité est clair à ce sujet. La nationalité d'origine s'acquiert de deux façons: En raison de la naissance au Niger "est 
tution et 6 du code électoral ne désignent que les Nigériens. Ce silence signifie que les Nigériens desquels il est question peuvent être des Nigériens d'origine ou des Nigériens par naturalisation.

\section{b) La condition de l'âge}

Il faut avoir 18 ans accomplis au jour du scrutin ou être mineur émancipé. "Le mineur est émancipé de plein droit par le mariage." "Le mineur qui aura atteint l'âge de seize ans révolus pourra être émancipé lorsqu'il exerce une profession séparée lui permettant de subvenir à ses besoins." Cette émancipation sera prononcée par le juge des tutelles à la demande soit des père et mère soit de l'un d'eux, soit du tuteur autorisé par le Conseil de famille. ${ }^{4}$ L'émancipation efface la condition d'âge de 18 ans et confêre au mineur qui n'a pas atteint cet âge là la jouissance du droit de vote.

\section{c) La jouissance des droits civils et politiques}

Les articles 8 de la Constitution et 6 du code électoral indiquent que le ou la Nigérienne doit jouir de ses droits civils et politiques. L'article 6 du code électoral ajoute que le citoyen ne doit pas se trouver dans les cas d'incapacité prévus par la loi. La déchéance des droits civils et politiques se rattache ici à une notion d'indignité. Elle frappe les personnes "dont le comportement passé ne permet pas de penser qu'elles pourraient remplir avec honneur et dignité leur devoir de citoyen". 5 Sont concernés par cette disposition:

- les individus condamnés pour crime et non réhabilités,

- les individus condamnés pour délit à une peine d'emprisonnement ferme égale ou supérieure à un an et non réhabilités,

- ceux qui sont en état de contumace,

- ceux qui sont déclarés en faillite et ayant fait l'objet d'une condamnation pour banqueroute frauduleuse et non réhabilités.

Nigérien tout individu né au Niger d'un ascendant direct au premier degré qui y est lui-même né ..." (article 8). "Est Nigérien l'enfant né au Niger de parents inconnus..." (article 10). En raison de la filiation "est Nigérien l'enfant né d'un père nigérien, l'enfant naturel, lorsque le père à l'égard duquel la filiation est établie est Nigérien (article 11). Peut opter jusqu'à la majorité pour la nationalité nigérienne à condition qu'il réside habituellement au Niger: "l'enfant légitime né d'une mère nigérienne et d'un père de nationalité étrangère, sans nationalité ou de nationalité inconnue; l'enfant naturel, lorsque la mère à l'égard de laquelle la filiation a été établie est nigérienne, si l'autre ascendant est de nationalité étrangère, sans nationalité ou de nationalité inconnue" (article 12). Cf. Titre II de l'Ordonnance no. 84-33 du 23 août 1994 portant code de la nationalité nigérienne.

L'Ordonnance no. 84-33 du 23 août 1994 portant code de la nationalité nigérienne soit par la filiation soit pa la naturalisation. Cf. Titre II de l'Ordonnance.

4 Cf. articles 464 et 465 du projet de code de la famille, version janvier 1993.

5 Cf. Pactet, Pierre, Institutions politiques et droit constitutionnel, Paris, 10 ème éd., p. 350.

6 Cf. Article 8 du code électoral. 
Les personnes frappées par le cas d'incapacité sont les internés, c'est-à-dire ceux qui ne sont pas sains d'esprit et qui ne peuvent par conséquent accomplir un acte valable dans la vie civile, et les interdits, c'est-à-dire les majeurs protégés par la loi ${ }^{7}$, les majeurs en tutelle $^{8}$, et les majeurs sous la sauvegarde de la justice ${ }^{9}$. Ces trois conditions réunies confêrent à l'individu la jouissance du droit de vote, encore faudrait-il qu'il en ait l'exercice.

\section{L'exercice du droit de vote}

Il est subordonné à l'inscription sur une liste électorale. Seuls les électeurs inscrits sur une liste électorale peuvent exercer, aux termes de l'article 7 du code électoral, leur droit de vote. Il en résulte une obligation tacite d'inscription sur les listes électorales. ${ }^{10}$ L'inscription sur les listes électorales se fait à certaines conditions; elle est subordonnée à une certaine procédure et le contentieux qui peut en résulter est réglé par le code électoral.

\section{a) Les conditions de l'inscription}

Elles sont au nombre de deux:

- il faut être électeur,

- l'électeur doit présenter avec la commune électorale sur la liste de laquelle il entend se faire inscrire un lieu qui résulte du domicile ou de la résidence.

Le domicile de tout personne est, quant à l'exercice de ses droits civils, le lieu de son principal établissement ou résidence.

\section{b) La procédure de l'inscription}

dans chaque arrondissement, commune, ambassade ou consulat, les listes électorales sont permanentes. ${ }^{12}$ Elles sont révisées chaque année du 1er octobre au 31 décembre par une

C'est-à-dire le majeur qu'une altération des facultés personnelles met dans l'incapacité de pourvoir seul à ses intérêts.

8 C'est-à-dire ceux qui du fait d'une altération de leur facultés personnelles ont besoin d'être représentés d'une manière continue dans les actes de la vie civile.

9 C'est-à-dire ceux qui du fait d'une altération de leur facultés personnelles sont soignés dans un hôpital, une maison de santé ou un établissement d'aliénés.

10 Les termes exacts de l'article 7 du code électoral sont: "Nul ne peut voter s'il n'est inscrit sur la liste électorale de son domicile ou de sa résidence".

11 Cf. Article 10, projet du code de la famille.

12 Cf. article 11 du code électoral. 
commission administrative. En cas de besoin les listes électorales peuvent exceptionnellement être modifiées.

\section{c) Le contentieux de l'inscription}

Il comporte un recours porté devant le juge de paix et devant la Cour Suprême. Le recours contre les décisions de la commission administrative ${ }^{13}$ est formé sur simple déclaration au greffe de la justice de paix. Sont admis à former un recours, les citoyens omis sur une liste électorale, les tiers inscrits s'ils veulent obtenir la radiation d'une personne indûment inscrite ou l'inscription d'une personne omise dans la même circonscription. ${ }^{14}$ "Dans les 10 jours qui suivent la dite déclaration, le juge de paix statue sans frais ni forme de procédure et sur simple avertissement donné trois jours au moins à l'avance à toutes les parties intéressées." ${ }^{15}$ La décision du juge de paix, rendue en dernier ressort, peut être déférée en cassation devant la Cour Suprême.

Le code électoral réprime certaines infractions parmi lesquelles on peut retenir celles se rapportant à des inscriptions frauduleuses qu'il punit d'amendes et de prison. ${ }^{16}$

L'inscription sur une liste électorale donne droit à la délivrance d'une carte d'électeur qui est personnelle. Elle est remise à l'électeur par l'autorité qui a dressé la liste électorale. ${ }^{17}$ Elle est exigée au moment de l'operation de vote.

Le vote par procuration est permis dans certaines conditions exceptionnelles définies par l'article 53 du code électoral. ${ }^{18}$

13 Elle statue sur les réclamations en inscription ou en radiation formulées dans un délai d'un mois à partir de l'affichage de l'avis d'établissement des listes (article 16 du code électoral). Elle dispose d'un délai de 5 jours après sa saisie pour rendre et notofier par écrit sa décision aux parties intéressées (article 17, code électoral).

14 Cf. articles 14 et 15 du code électoral

15 Cf. article 18 alinéa 3 du code électoral.

16

L'article 129 du code électoral indique que: "toute personne qui se sera fait inscrire sur une liste électorale sous de faux noms ou de fausses qualités ou aura réclamé et obtenu une inscription sur deux ou plusieurs listes, sera punie d'un emprisonnement de deux mois à deux ans et d'une amende de vingt

17 mille francs à deux cent mille francs".

Cf. articles 29 et 5 du code électoral.

18 Cet article indique que le vote par procuration est admis en cas d'incapacité physique ou d'empêchement majeur; le mandataire, porteur d'une procuration, doit être électeur dans le même bureau de vote que le mandat. Il ne peut porter plus d'une procuration uninominale. La procuration doit être légalisée par une autorité administrative, judiciaire, consulaire ou diplomatique et doit porter la signature du mandat et le sceau de l'autorité ayant procédé à la législation. Cet article a été précisé par le ministre de l'intérieur. 


\section{B. Les élections}

Les textes fondamentaux de la 3ème République nigérienne distinguent plusieurs sortes d'élections: les élections politiques qui sont celles concernant le Président de la République, les députés à l'Assemblée Nationale et les conseillers d'arrondissement ou municipaux, et le référendum qui est la consultation par vote du peuple pour approuver ou rejeter une mesure proposée par les pouvoirs publics. ${ }^{19}$ Ces textes ayant été appliqués pour la première fois, nous suivrons l'ordre chronologique de leur organisation. ${ }^{20}$

\section{Les élections législative}

L'article 67 de la Constitution du 26 décembre 1992 indique que: "les Députés sont élus au suffrage universel, libre, direct, égal et secret..., la durée de la législature est de cinq ans..., la loi fixe le nombre des membres de l'Assemblée Nationale, leur indemnité, les conditions d'éligibilité, le régime des inéligibilités et des incompatibilités, les modalités de scrutin ainsi que les conditions dans lesquelles il y a lieu d'organiser de nouvelles élections en cas de vacance du siège des députés". Le code électoral règlemente tous ces domaines laissés à la loi par la Constitution.

\section{a) L'éligibilité et les candidatures}

\section{aa) L'éligibilité}

Pour être éligible, il faut être électeur, c'est-à-dire satisfaire aux conditions que nous avons évoquées ci-dessus. ${ }^{21}$ Le code électoral ${ }^{22}$ indique que "sont éligibles à l'Assemblée Nationale les citoyens nigériens agés de 25 ans révolus au jour du scrutin et n'entrant dans aucun des cas d'incapacité prévu à l'article 8 du présent code".

Le code établit des inéligibilités relatives pour certaines personnes nommément désignées lorsqu'elles exercent certaines fonctions, à moins que leur démission ou leur disponibilité ne soit acquise régulièrement trois mois avant l'ouverture officielle de la campagne électo-

\section{9}

20

21

22

Cf. article 3 du code électoral.

Le référendum constitutionnel, première opération électorale organisée dans le cadre de la 3ème République a été étudié supra, cf. introduction.

L'article 6 du code électoral indique que: "sont électeurs les Nigériens des deux sexes âgés de 18 ans accomplis au jour du scrutin ou mineurs émancipés jouissant de leurs droits civiques et politiques et n'étant dans aucun des cas d'incapacité prévu par la loi".

Cf. article 92 . 
rale. ${ }^{23}$ Il retient également les inéligibilités absolues, c'est-à-dire, en conformité avec la non-acquisition de la qualité d'électeur, celles qui résultent d'une certaine indignité attestée par une condamnation pénale ou une décision judiciaire. ${ }^{24}$

\section{bb) Les candidatures}

Avant le dépôt des candidatures, un cautionnement électoral doit être versé au trésor public. Il est remboursé aux candidats et aux listes qui obtiennent au moins cinq pour cent des suffrages exprimés. ${ }^{25}$ Le remboursement est exigible au moins trente jours après la proclamation définitive des résultats. ${ }^{26}$

Une déclaration de candidature est nécessaire, elle doit remplir certaines conditions. ${ }^{27}$

Elle doit être déposée en trois exemplaires pour enregistrement, affichage et diffusion à la préfecture dont dépend la circonscription électorale. Le préfet envoie un exemplaire à l'autorité de la circonscription électorale concernée et un autre au ministère de l'intérieur.

La Cour Suprême statue sur l'éligibilité des candidats. Cependant, le code électoral est muet sur l'autorité qui est habilitée à saisir la Cour Suprême et sur les modalités dans lesquelles elle doit se prononcer. On peut penser que c'est le préfet, l'autorité de la circonscription électorale concernée ou le Ministre de l'intérieur qui, s'ils ont un doute sur l'éligibilité des candidats, saisissent la Cour Suprême.

Avec le scrutin de liste, les candidats font une déclaration collective. Chaque candidat a un suppléant personnel qui figure sous cette appellation sur la liste. "La déclaration de candidature doit comporter l'indication de la circonscription électorale dans laquelle est présentée la liste des candidats." 28 "Seuls les partis politiques légalement constitués sont admis à déposer une liste de candidats et une seule dans le cas du scrutin de liste." 29

23 Sont obligés de démissioner, les militaires des forces armées et de la gendarmerie, les chefs traditionnels, les préfets et les sous-préfets. Doivent prendre une disponibilité, les magistrats de l'ordre judiciaire en activité et les agents de force de sécurité intérieure: police, garde républicaine et garde présidentielle. Cf. articles 8 et 92 du code électoral.

Il est de 2.000.000 de FCFA pour l'élection du président de la République et de 100.000 FCFA pour l'élection des députés.

Cf. article 36 du code électoral.

Elle doit être légalisée et comporter un certain nombre d'informations (cf. article 28 du code électoral).

Cf. article 29 du code électoral, alinéa 3.

Cf. article 31 du code électoral. 


\section{b) Le mode de scrutin}

Aux termes de l'article 88 du code électoral, "les députés sont élus au scrutin de liste à un tour avec représentation proportionnelle selon le plus fort reste...".La représentation proportionnelle est un système électoral qui s'efforce d'assurer la représentation de tous les partis groupant un nombre minimum de suffrage: Chaque liste doit recevoir un nombre d'élus proportionnel au nombre de voix qu'elle a recueillies.

Dans ce mode de scrutin, la répartition des sièges se fait en deux temps: Lors d'une première répartition des sièges, plusieurs procédés peuvent être utilisés. ${ }^{30}$ Le code électoral nigérien a retenu le quotient par circonscription ou quotient électoral. En effet, selon l'article 88 alinéa 2, "l'attribution des sièges selon la représentation proportionnelle ... consiste à attribuer autant de sièges à une liste que le nombre de ses suffrages contient le quotient électoral. Le quotient électoral est le résultat de la division des suffrages exprimés par le nombre de sièges à pouvoir dans une circonscription électorale". Lors d'une seconde répartition des sièges, on utilise selon le code électoral nigérien la règle du plus fort reste ${ }^{31}$ en vertu de laquelle on attribue le ou les sièges restants à la ou aux listes qui ont le plus grand nombre de suffrages inemployés. ${ }^{32}$

\section{Illustration de la méthode retenue par le code électoral nigérien}

Soit une circonscription électorale où il y a 100.000 suffrages exprimés. Il y a 5 sièges à pourvoir. On cherche le quotient électoral:

$$
\left(\mathrm{QE}=\frac{100.000}{5}=20.000\right)
$$

30

Il y a celui du nombre uniforme dans lequel on détermine, avant le scrutin, pour l'ensemble du territoire, le nombre de voix nécessaires pour qu'une liste ait droit à un siège: Chaque liste aura autant de sièges que le montant des suffrages recueillies par elle contiendra le nombre uniforme. Il y a le quotient national qui consiste à diverser, après les élections, le nombre de suffrages exprimés de l'ensemble du territoire par le nombre national de sièges à pourvoir. Le résultat ainsi obtenu est le quotient national que l'on utilise après comme nombre uniforme selon le même mécanisme. Il y a enfin le quotient électoral.

31 Il faut souligner qu'ici aussi, il y a deux méthodes utilisées: On peut regrouper les voix restantes au plan national. L'addition des restes obtenus par les différentes listes d'un même parti est divisée par le nombre uniforme pour déterminer le nombre d'élus. On peut, lorsque comme c'est le cas au Niger ou c'est le quotient électoral qui est utilisé, répartir les voix restantes par circonscription selon deux méthodes: le procédé de la plus forte moyenne qui consiste à diviser pour chaque liste le nombre total de suffrages recueillis, par le nombre de sièges qu'on lui a déjà attribué plus 1 . Chaque liste obtient alors une certaine moyenne; c'est la liste qui a la plus forte moyenne qui emporte le siège.

Il faut noter que ce système favorise les petits partis qui, sans obtenir le quotient, réussissent parfois un reste assez important. 
On imagine que:

la liste $\mathrm{A}$ a obtenu 36.000 voix

la liste B " " 25.000 voix

la liste $C " \quad$ " 19.000 voix

la liste D " " 11.000 voix

la liste $\mathrm{E} " \quad$ " 9.000 voix

Après la lère répartition des sièges:

A obtient $(36.000: 20.000=) 1$ siège, reste de 16.000 voix

B obtient $(25.000: 20.000=) 1$ siège, reste de 5.000 voix

C obtient (19.000: $20.000=) 0$ siège, reste de 19.000 voix

D obtient $(11.000: 20.000=) 0$ siège, reste de 11.000 voix

E obtient ( $9.000: 20.000=) 0$ siège, reste de 9.000 voix

On procède à la seconde répartition des sièges (au plus fort reste)

la liste $\mathrm{C}$ obtient 1 siège,

la liste $\mathrm{A}$ obtient 1 siège,

la liste $\mathrm{D}$ obtient 1 siège.

Au total:

liste $\mathrm{A}=2$ sièges

liste $\mathrm{B}=1$ siège

liste $\mathrm{C}=1$ siège

liste $\mathrm{D}=1$ siège

\section{c) Les circonscriptions électorales}

"Pour l'élection des députés, la circonscription électorale est le département ou la communauté urbaine. Une loi détermine le nombre de sièges à pourvoir par département et par communauté urbaine en fonction du nombre des arrondissements et communes et de leurs poids démographique." ${ }^{33}$ C'est sur la base de ces dispositions que le nombre des députés a été fixé à $77 .^{34}$ Département de Dosso 10 sièges, Département de Maradi 13 sièges, Département de Tillaberi 13 sièges, Département de Tahona 13 sièges, Département de Zinder 14 sièges, Communauté Urbaine de Niamey 4 sièges. 
Pour pallier en partie les inconvénients de la représentation proportionnelle ${ }^{35}$ et pour remédier certaines particularités régionales, il a été crée 8 circonscriptions spéciales. Le mode de scrutin dans ces circonscriptions est le scrutin majoritaire uninominal à un tour. ${ }^{36}$ En effet, il s'agit de petites circonscriptions à un seul siège, est élu le candidat qui recueille le plus de voix.

Cette combinaison entre la représentation proportionnelle et le scrutin majoritaire annule les effets de justice recherchés avec l'adoption du scrutin de liste. Aux élections législatives du 14 février 1993, les premières librement et démocratiquement organisées depuis l'accession du Niger à l'indépendande, douze partis ${ }^{37}$ présentèrent 599 prétendants. Au total 83 sièges étaient à pourvoir.

\section{d) Le régime des élections}

\section{aa) La campagne législative}

Elle s'ouvre un mois avant le scrutin et est close l'avant-veille à minuit. Le code électoral règle les questions relatives au ballotage (en cas d'élection présidentielle), à la propagande électorale qui se fait par affiches, distribution de circulaires, réunions, voies de presse et autres manifestations culturelles. Il pose les conditions de validité de toute réunion électorale $^{38}$ qui ne doit pas être de nature à troubler l'ordre public. Le code électoral pose enfin certains interdits.

En effet, la représentation proportionnelle multiplie le nombre des partis en incitant les petits partis à se maintenir même s'il ne jouent pas un rôle décisif; elle dépossède l'électeur de sa souveraineté et la transfère aux partis car ce sont ces demiers qui élaborent les listes et qui s'assurent de faire élire leurs dirigeants en les désignant "tête de liste"; enfin, elle enlève une partie de son intérêt à la procédure de dissolution que detient le Président de la République car elle cristallise les positions des différents partis au sein de l'assemblée, ce qui ne favorise pas l'alternance.

Il s'agit de l'arrondissement de Bilma, du poste administratif de Bankilaré, du poste administratif de Bermo, du poste administratif de N'gourti, du poste administratif de Tassara, du poste administratif de Temker et du poste administratif de Torodi qui se voient attribuer chacun un siège.

L'ANDP Zaman Lahya avec 78 candidats, le CDS Rahama avec 79 candidats, le MNSD Nassara avec 83 candidats, le PNDS Tarraya avec 83 candidats, le RDA avec 18 candidats, le PMT Albarka avec 55 candidats, le PUND Salama avec 4 candidats, le PSDN Alhéri avec 24 candidats, l'UDPS Amana 1 candidat et l'UDP-Alkawali 14 candidats.

Selon l'article 66 du code électoral, toute réunion électorale est soumise à l'obligation d'une déclaration préalable auprès de l'autorité de la circonscription administrative dans le ressort de laquelle se trouve le lieu où elle doit se tenir. La déclaration est effectuée au moins six heures avant la tenue effective de la réunion.

En effet, aux termes de l'article 67, les propagandes, affiches, harangues, sermons et professions de foi à caractère religieux sont interdits. Les tracts, les déclarations et harangues à caractère diffamatoire ou injurieux à l'égard des autres candidats sont interdits. Cet article interdit également les harangues, déclarations, sermons et professions de foi à caratère régionaliste, ethnique ou racial etc... L'utilisation des moyens de l'Etat à des fins de propagande etc... 


\section{bb) Le contentieux électoral}

Il est confié à la Cour Suprême. La réclamation doit être adressée au Président de la Cour Suprême au plus tard le 15ème jour suivant la proclamation définitive des résultats par tout électeur (qui peut demander la nullité des opérations électorales de son bureau de vote) et par tout parti politique qui a présenté des candidats (il faut demander alors soit lui-même, soit par son délégué, l'annulation des opérations de la circonscription où il a déposé sa candidature).

On peut légitimement estimer que la Cour Suprême peut s'auto-saisir, à l'occasion de ses vérifications, des réclamations signalées par les électeurs ou par les représentants des candidats auprès des commissions départementales d'élection, à la condition que ces réclamations aient été inscrites sur les procès-verbaux de dépouillement.

C'est la chambre constitutionnelle de la Cour Suprême qui est seule compétente pour statuer sur les réclamations. Elle juge en premier et dernier ressort. Elle peut annuler totalement ou partiellement les opérations de vote. ${ }^{40}$ C'est sur cette base que la Cour Suprême a annulé les opérations de la circonscription spéciale de Tesker ${ }^{41}$ et celles de 9 bureaux de vote de la ville de Zinder.

Les élections législatives organisées le 14 février 1993, avec un taux de participation de $33,2 \%$, ont donné les résultats suivants:

$\begin{array}{lc}\text { MNSD Nassara } & 29 \text { sièges } \\ \text { CDS Rahama } & 22 \text { sièges } \\ \text { ANDP Zaman-Lahya } & 11 \text { sièges } \\ \text { PNDS Tarraya } & 11 \text { sièges } \\ \text { PPN/RDA } & 2 \text { sièges } \\ \text { PSDN Alhéri } & 1 \text { siège } \\ \text { UDFP Sawaba } & 2 \text { sièges } \\ \text { UPDP Chamoua } & 2 \text { sièges } \\ \text { UDPS Amana } & 1 \text { siège }\end{array}$

40

Selon l'article 78 du code électoral, constituent des causes d'annulation des élections, la constatation de l'inéligibilité d'un candidat, l'existence d'une candidature multiple, le défaut d'isoloir dans un bureau de vote même hors de toute intention de fraude, la violence, la fraude, la corruption faussant le résultat du scrutin pour l'élection des candidats, la participation à la propagande électorale par des actes ou des déclarations réprimés conformément aux dispositions pénales du code électoral.

41

C'était le 18 mars 1993, à l'occasion de la proclamation des résultats définitifs des élections législatives du 14 février 1993. La cour a annulé toutes les opérations de la circonscription spéciale de Tesker où de nouvelles élections seront organisées dans un délai de 40 jours. Elles ont été organisées le 11 avril 1993.

42

La cour a annulé un siège d'une circonscription spéciale. Tesker détenu par ce partie. 
Les élections législatives ont consacré la percée électorale du MNSD-Nassara issu de l'ancien parti-Etat. ${ }^{43}$

C'est pour "barrer la route" au MNSD que certains leaders de partis politiques ${ }^{44}$ ont signé un accord, l'Alliance des Forces du Changement (AFC) ${ }^{45}$ qui est un front commun de lutte sur la base d'une plate-forme commune. L'objectif premier est de constituer un groupe parlementaire majoritaire. En effet, l'addition des sièges obtenus par les partis composant l'alliance donne à l'AFC la majorité à l'Assemblée Nationale. L'AFC, a fait du mot "changement" son cheval de bataille mais les contours de celui-ci ne sont pas clairs. ${ }^{46}$

La première Assemblée Nationale de la 3ème République du Niger fut installée le vendredi 9 avril 1993.

\section{Les élections présidentielles}

Selon l'article 38 de la Constitution du 26 décembre 1992, "le Président de la République est élu pour cinq ans au suffrage universel, libre, direct, égal et secret. Il n'est rééligible qu'un fois". L'article 40 alinéa 2 ajoute que: "La loi précise les conditions d'éligibilité, de présentation des candidatures, de déroulement du scrutin, de dépouillement et de proclamation des résultats".

Cette victoire est due à l'échec de l'après-conférence Nationale. En effet, pendant ce forum il s'agissait coûte que coûte de balayer l'ancien ordre pour le remplacer par un nouveau. Des hommes ont été triés sur le volet pour "redresser la barre" et conduire les 15 mois de transition. Selon Mr. Hima "ils étaient plutôt préoccupés par les luttes d'influence. Nombre d'entr'eux se sont attelés à préparer l'après-transition soit pour eux-mêmes soit pour leur parti politique. On est au gouvernement et on est en même temps dans l'opposition contre son ... gouvernement. ... Quinze mois de transition ne leur ont pas suffit à assimiler et maîtriser leurs cours pour convaincre les examinateurs..." Cf. interview Sahel-Dimanche no. 509 du 19 février 1993, p. 7 .

Un collectif de 9 partis, l'ANDP, la CDS, le PNDS, PPN-RDA, PSDN, PUND, WDP, UDPS, PRLN. Conclue le mardi 16 février 1993.

Tawaya, Batouré, Cris et chuchotements, dans: Le démocrate du Lundi, 15 mars 1993, p. 4, se pose la question suivante: Quel crédit peut-on accorder au mot changement lorsque les porte-drapeaux et thuriféraires n'hésitent pas à construire la stratégie de leur combat d'aujourd'hui et probablement leur victoire de demain sur le mépris souverain des dispositions d'un code électoral qu'ils ont contribué à élaborer et à faire adopter?" L'auteur a préalablement relevé que les chantres du changement ont d'abord foulé au pied la Constitution du 26 décembre 1992 (en nommant le Premier Ministre et le Président de l'Assemblée Nationale alors que la Constitution a clairement indiqué les autorités compétentes pour le faire) et le code électoral (en violant l'article 138 qui interdit et punit toute promesse d'emplois de l'Etat ou de ses démembrements à des fins électoralistes). 


\section{a) L'éligibilité et les candidatures}

\section{aa) L'éligibilité}

Aux termes de l'article 38 alinéa 2 de la Constitution ${ }^{47}$ "est éligible à la présidence de la République tout Nigérien, de nationalité d'origine, âgé de quarante ans au moins et jouissant de ses droits civils et politiques". Comme pour les candidats aux élections législatives, les candidats aux élections présidentielles doivent être électeurs et ne doivent pas tomber sous le coup de l'inéligibilité relative de l'article 81 du code électoral ni de l'inéligibilité absolue de l'article 8 du même code. ${ }^{48}$

\section{bb) Les candidatures}

Les déclarations de candidatures se font dans les mêmes conditions que celles des prétendants aux sièges à l'Assemblée. Cependant, pour les présidentielles, les déclarations de candidature sont déposées en deux exemplaires au ministère de l'intérieur cinquante jours au moins avant le jour du scrutin. Seuls les partis politiques légalement constitués peuvent présenter des candidats; chaque partie politique ne peut présenter qu'un seul candidat.

"Quarante-cinq jours avant l'ouverture du scrutin, le ministre chargé de l'intérieur arrête la liste des candidats et la transmet à la Cour Suprême qui dispose d'un délai de 48 heures pour se prononcer sur l'éligibilité des candidats." ${ }^{49}$ En cas de décès d'un candidat ou de survenance d'une cause d'inéligibilité absolue en cours de campagne, le parti politique concerné remplace le candidat inéligible par un nouveau candidat. Dans ce cas, le ministre de l'intérieur transmet la nouvelle candidature dans un délai de 24 heures à la Cour Suprême qui doit se prononcer dans les 24 heures sur l'éligibilité du remplaçant.

\section{b) Le mode de scrutin}

Selon les dispositions de l'article 39 de la Constitution nigérienne, "l'élection du Président de la République a lieu au scrutin majoritaire à deux tours". Dans ce système est déclaré élu le candidat qui obtient, au premier tour, la majorité absolue (plus de la moitié des suffrages exprimés). Si tel n'est pas le cas, un second tour est organisé à la suite duquel est élu celui qui obtient le plus grand nombre de voix (majorité relative). Le second tour doit être organisé 14 jours après et seuls les deux candidats arrivés en tête lors du premier tour prennent part au scrutin. 50

47

48

49

50

Et de l'article 80 du code électoral.

Voir développements supra note 12 .

Cf. article 84 du code électoral.

Cf. article 39 alinéa 2 et 3 de la Constitution et article 86 alinéa 1 et 2 du code électoral. 
Cette disposition a failli poser des problèmes lors du premier tour des élections présidentielles organisé le 27 février 1993. Vu les différents problèmes matériels, la date du second tour a été reportée par les autorités qui ont procédé à une interprétation ${ }^{51}$ des articles 39 de la Constitution et 86 du code électoral en faisant courir le délai de 14 jours à partir de la proclamation des résultats définitifs par la Cour Suprême. Cette dernière enterina tacitement cette interprétation et le deuxième tour des élections présidentielles fut fixé au 27 mars 1993. Il opposa les deux candidats arrivés en tête à savoir le candidat du MNSD Nassara, Mr Tandja Mamadou (qui a recueilli $34 \%$ des voix) au candidat de la CDS Rahama, Mr Mahamane Ousmane (avec $26 \%$ des voix).

\section{c) Les circonscriptions électorales}

C'est le territoire national étendu aux missions diplomatiques et consulaires. ${ }^{52}$

\section{d) Le régime des élections}

\section{aa) La campagne présidentielle}

Elle est soumise aux mêmes règles que la campagne législative. Toutefois, en cas de ballotage, la campagne est à nouveau ouverte dès la proclamation des résultats du premier tour; elle est close l'avant-veille à minuit.

\section{bb) Le contentieux électoral}

Il obéit aux mêmes dispositions que celui de la campagne législative. Le premier tour des élections présidentielles (taux de participation 32,59\%) a posé le problème de l'interprétation de l'article 64 du code électoral en vertu duquel "seuls les partis politiques légalement constitués et leurs candidats régulièrement inscrits sont autorisés à organiser des réunions électorales".

En effet, les leaders des partis composant l'Alliance de Forces du Changement (AFC), constituée au lendemain des élections législatives, ont soutenu qu'ils pouvaient battre campagne ès-qualité pour le dirigeant du parti CDS-Rahama en lice pour le second tour. Le MNSD Nassara quant à lui a soutenu que seul le parti véritablement en lice avait, selon les termes du code électoral, le droit de battre campagne.

La Cour Suprême, saisie par le Haut Conseil de la République en vue de l'interprétation de l'article en question a estimé que: "L'Alliance des Forces du Changement (AFC) ne pouvait battre campagne ès-qualité, étant un groupement circonstanciel de fait comparable

51

Ces autorités n'avaient pas compétence pour le faire, seule la Cour Suprême est habilitée à procéder à l'interprétation des textes fondamentaux.

52

Cf. article 26 alinéa ler du code électoral. 
à un comité de soutien ordinaires. ${ }^{53}$ Aussi Mahamane Ousmane demeure-t-il le candidat de la CDS-Rahama ${ }^{54}$, à qui l'AFC apporte son soutien sous la houlette de la CDSRahama". Les parties concernés ont fait fi de cet avis de la Cour Suprême en battant campagne sous la banière de l'AFC aux couleurs de la CDS.

L'AFC a par ailleurs procédé à la répartition des postes de Premier Ministre ${ }^{55}$ et de Président de l'Assemblée Nationale ${ }^{56}$ au cas ou son candidat serait élu Président. Cette répartition des postes a donné lieu à la saisie de la Cour Suprême sur la base de la violation de l'article 138 du code électoral qui indique que: "Quiconque, par des dons ou libéralités en argent ou en nature, par des promesses d'emplois de l'Etat, de ses démembrements ou des entreprises publiques, faits en vue d'influencer le vote d'un ou de plusieurs électeurs, aura obtenu ou tenté d'obtenir leur suffrage soit directement soit par l'entremise d'un tiers, quiconque, par les mêmes moyens aura déterminé ou tenté de déterminer un ou plusieurs d'entre eux à s'abstenir, sera puni d'un à cinq ans d'emprisonnement et d'une amende de cinquante mille à cinq cent mille francs..." 57

La Cour ne s'est prononcée sur la violation de l'article 138 qu'au moment de la validation des résultats du second tour des élections présidentielles ${ }^{58}$ en rejettant la demande du MNSD. Pour statuer, la Cour a saisi le parquet de Niamey afin de connaître la suite à réserver à la plainte du MNSD-Nassara. Le Procureur de la République près le tribunal de Niamey a transmis ${ }^{59}$ à la Cour Suprême un avis de classement de la plainte sans suite pour inopportunité de poursuites.

Les enseignements qu'on peut tirer après le premier tour des élections est que le manque d'autorité de l'Etat a permi à la démocratie nigérienne de démarrer sur des bases fausses faites de violations du texte constitutionnel et du code électoral et du silence des autorités qui devaient intervenir pour rappeller les protagonistes à l'ordre. Quel que soient les

La cour s'est fondée sur l'article 32 du code électoral qui indique les conditions de validité des groupements de partis et l'AFC ne réunissait pas ces conditions. En effet selon ledit article "les partis politiques peuvent se concerter pour présenter une liste commune de candidats. Dans ce cas, la liste commune doit porter en en-tête la désignation des partis concernés et mentionner pour chaque candidat son appartance politique personnelle".

Au lendemain du premier tour des élections, les chefs de file de l'AFC avaient indiqué qu'à partir du jour de leur déclaration, Mr Mahamane Ousmane cessait d'être le candidat de son parti et devenait celui de l'AFC. Or cette dernière n'est pas un parti légalement reconnu et constitué ce qui disqualifiait le candidat en question.

Attribué au candidat de l'ANDP.

56

Attribué au candidat de l'ANDP.

57 Selon l'article 78 du code électoral, ces faits constituent une cause d'annulation des élections.

58

Le jeu de 8 avril 1993, alors qu'elle aurait dû le faire avant le 2ème tour.

59

Le 6 avril 1993 
motivations des autorités en question ${ }^{60}$, il est nécessaire en démocratie véritable que la loi soit appliquées dans toute sa rigueur toutes les fois où elle est violée.

Le second tour des élections présidentielles (taux de participation 35,3\%) fut organisé le 27 mars 1993. Le fait majeur de celui-ci est la victoire du candidat de la CDS-Rahama soutenu par l'Alliance des Forces du Changement avec 54,42\% des suffrages exprimés. Cette victoire est due à un report parfait des voix des autres candidats. ${ }^{61}$ Le report n'a été possible que suite à la répartition des postes ci-dessus relevée. ${ }^{62}$

\section{Les partis politiques}

\section{Statut des partis politiques}

C'est l'acte Fondamental no. XXIV/CN du 3 novembre 1991 appelé "Charte des partis politiques" qui définit et précise le statut des partis politiques. ${ }^{63}$ Il s'agit d'un ensemble de règles dont l'objet est d'organiser la formation et le fonctionnement des partis politiques.

La Charte des partis impose à ces derniers de proscrire dans leurs activités le régionalisme, le racisme, l'incitation et le recours à la violence sous toutes ses formes. Tout Nigérien jouissant de ses droits civils et politiques peut adhérer au parti politique de son choix. ${ }^{64}$ Cependant, de par leurs fonctions, certaines personnes ne peuvent militer dans aucun parti politique; il s'agit des magistrats, des éléments des forces armées et de sécurité, les cadres de commandement, les chefs traditionnels etc.

Pour être dirigeant d'un parti politique, il faut, selon les termes de la Charte des partis, avoir la nationalité nigérienne, avoir 18 ans au moins, jouir de ses droits civils et politiques, n'avoir pas été condamné à une peine affictive ou infamante et avoir son domicile ou résidence sur le territoire national.

60

Maintien de l'ordre public, car une application stricte de la loi pouvait y porter atteinte.

Le candidat de la CDS a amélioré son score dans tous les départements. Le taux de participation s'est également nettement amélioré $(35,22 \%$ contre $32 \%$ au premier tour). Le candidat du MNSD a, quant à lui, recueilli $45,59 \%$ des voix.

Beaucoup d'électeurs ont soutenu que si le leader de leur parti ne bénéficiait pas d'un poste, ils n'auraient pas voté pour le candidat de la CDS.

La Charte définit les partis politiques en son article ler comme des associations à but non lucratif qui conformément à la Constitution regroupent des citoyens nigériens autour d'un projet de société et d'un programme politique en vue de concourir à l'expression du suffrage universel et de participer à la vie politique par des moyens démocratiques et pacifiques.

Cf. article 6 de la Charte des partis. 
Un parti politique n'obtient sa capacité juridique qu'après avoir effectué certaines formalités. ${ }^{65}$ La charte précise que les activités des partis sont financées par les cotisations des membres, le produit des ventes des cartes, les dons, les legs, les revenus liés à leurs activités, les subventions et aides éventuelles de l'Etat. ${ }^{66}$ Cependant, la charte pose une série de limites; les dons et les libéralités perçues devront faire l'objet d'une déclaration au ministère de l'intérieur. Le montant de ces dons et libéralités provenant de l'extérieur pour le compte d'un parti ne doivent pas dépasser $20 \%$ du montant total des ressources dudit parti. La limite est de $50 \%$ pour les dons provenant des personnes physiques ou morales nationales. La subvention étatique sera proportionnelle au nombre de députés inscrits pour chaque parti à l'Assemblée Nationale.

La charte oblige les partis à tenir une comptabilité et un inventaire de leurs biens et à présenter leurs comptes annuels au ministère des finances. Les partis politiques peuvent être suspendus de leurs activités en raison de violations graves des lois en vigueur. La suspension ne peut excéder trois mois. Le ministère de l'intérieur peut demander la dissolution d'une formation politique par voie judiciaire.

Il faut enfin relever la psychose des coups d'Etat qui se matérialise dans la Charte par l'article 29 qui fait encourir à tout dirigeant d'un partie qui par ses écrits, déclarations publiques, démarches incite ou invite les forces de sécurité à s'emparer du pouvoir d'Etat, une peine de 5 à 10 ans et une amende de 500.000 FCFA à 1.000.000 FCFA sans préjudice de la dissolution du parti concerné et de la confiscation de ses biens.

\section{Les principaux partis}

Il y a 18 partis politiques légalement reconnus au Niger. On ne retiendra ici que ceux qui ont obtenu des résultats substantiels aux législatives du 14 février $1993 .{ }^{67}$

65

Le dossier qui sera constitué doit être déposé au ministère de lintérieur. Il devra comprendre plusieurs éléments (liste des dirigeants, statut, dénomination et adresse). Après un contrôle de conformité, le Ministère de l'intérieur prend un arrêté autorisant le parti en question à exercer ses activités. Il est publié au Journal Officiel. Lorsqu'un parti n'obtient pas sa reconnaissance, il peut saisir la chambre administrative de la Cour Suprême dans les quinze jours qui suivent la notification. La cour statue selon la procédure d'urgence dans les 30 jours.

CF. article 16 de la Charte des partis.

67 Les autres partis politiques sont de moindre importance. Trois d'entr'eux comptent chacun deux députés. Ce sont: l'UDFP Sawaba, l'UPDP Chanova et le PNN-RDA. 


\section{a) Le Mouvement National pour la Société de Développement (MNSD-Nassara)}

Le Mouvement National pour la Société de Développement (MNSD-Nassara) ${ }^{68}$ a été, dans sa version actuelle, créé le 12 mars 1991. Avant le MNSD-Nassara existait le MNSD partie-Etat. Son emblème représente une plante en essor dont les racines venant des régions du pays convergent en direction du réceptacle dans lequel croît la plante. La floraison en ombelle matérialise d'une part la jeunesse, d'autre part les Producteurs Ruraux ainsi que les autres structures de participation de la Société de Développement dont les efforts conjugués viennent féconder le réceptacle qui symbolise la Patrie Nigérienne. Son signe de ralliement est les deux pouces levés, les autres doigts de la main fermés.

Son président aux élection présidentielles est Tandja Mamadou. Ses couleurs sont le vert et le rouge (parfois orange), sa devise "consultation, concertation, participation", et son idéologie est libérale. Le projet de société qu'il propose ${ }^{69}$ est la société de développement - un développement à la base qui fait une place de choix aux composantes essentielles de la population (les femmes, la jeunesse, le monde rural, l'enseignement, la santé etc.). La mise en oeuvre de cette société de développement passe d'abord par une véritable stratégie de réhabilitation nationale basée sur d'abord la restauration de l'autorité de l'Etat, ensuite le rétablissement de la confiance entre le citoyen et la puissance publique, c'est-à-dire remettre les Nigériens au travail, la restauration de la crédibilité et de l'image de marque du Niger, l'engagement ensuite du pays sur les rails du redressement économique, la décentralisation etc.

Ses structures comportent un Congrès qui est l'instance suprême du Parti, un Comité Central, la plus haute instance du Parti, organe de direction, de conception et d'animation du Parti, le Bureau Politique, organe d'exécution des décisions du Comité Central, les sections qui regroupent l'ensemble des sous-sections d'un Département ou d'une Communauté Urbaine, la Conférence Régionale, Instance Suprême de la section, la Conférence Sous Régionale, instance suprême de la sous-section, des Comités Locaux avec à leur tête la Conférence Locale et des Comités de Base coiffés par des Assemblées du Comité de base. Le MNSD-Nassara a bénéficié de l'organisation de l'ancien parti-Etat, ce qui lui garantit une très bonne implantation dans toutes les couches sociales du pays.

68

9 Qui fut l'essentiel du discours programme du Président Tandja Mamadou, candidat du parti aux élections présidentielles cf. MNSD Nassara, Discours Programme du Président du MNSD-Nassara, Elections Présidentielles 1993. 


\section{b) La Convention Démocratique et Sociale (CDS-Rahama)}

La Convention Démocratique et Sociale (CDS-Rahama) ${ }^{70}$ a été créée le 5 janvier 1991 et reconnue le 10 février 1992. Son emblème est une clé tendue par la main droite symbolisant l'ouverture et la liberté. Son signe de ralliement est le signe de la victoire à l'aide de l'index et du majeur levés en "V". Son président et candidat aux élections présidentielles est Mahamane Ousmane. Sa couleur est le vert symbolisant l'espoir, sa dévise "Unité Travail - Justice", et son idéologie est la voie sociale démocrate de développement.

Le projet de société que le parti propose est la création d'un modèle nigérien basé sur la construction d'une économie où coexistent les secteurs étatiques, semi-étatique et privé. Ses structures comportent un bureau national de 50 membres, une délégation nationale de 210 membres, 8 délégations régionales, 70 délégations sous-régionales, 102 délégations locales et plus de 600 cellules de base.

\section{c) Le Parti Nigérien pour la Démocratie et le Socialisme (PNDS-Tarayya)}

Le Parti Nigérien pour la Démocratie et le Socialisme (PNDS-Tarayya) ${ }^{71}$ a été créé en mars 1992. Mahamadou Issoufou est à la fois leader et candidat du parti aux élections présidentielles. L'emblème du PNDS est un cercle comprenant une roue dentelée symbolisant le progrès, une poignée de main en signe d'union et de solidarité, des épis de mil et de maïs et une tête de taureau représentant le monde rural. Le signe de ralliement est représenté par deux bras levés, les poings fermés. La couleur du parti est le rose, signe d'espoir, et son idéologie le socialisme démocratique. Les structures du parti sont le comité exécutif national (36 membres), 8 fédérations, 46 sections, 200 sous-sections et 8.000 districts.

\section{d) L'Alliance Nigérienne pour la Démocratie et le Progrès (ANDP Zaman-Lahiya)}

L'Alliance Nigérienne pour la Démocratie et le Progrès (ANDP Zaman-Lahiya) ${ }^{72}$ a été créée en février 1992 par le Colonel en retraite Moumouni Djermakoye Adamou, ancien ministre d'Etat, ancien ambassadeur. Le parti a été reconnu le 27 février 1992. Le signe de ralliement du parti est symbolisé par les deux mains entrelacées, les pouces en avant, symbole de l'unité. Son emblème est la carte du Niger sur fond d'un mur en brique (symbole de la construction nationale) avec comme couleurs de haut en bas: l'orange, le blanc et le vert. Sa devise est "un Peuple, un But, une Nation". Ses structures sont un 
comité exécutif national de 79 membres, 8 sections, 50 sous-sections, plusieurs cellules locales et de base. Son idéologie est le libéralisme économique contrôlé.

Le projet de société proposé par le Zaman Lahya est la défense des idéaux tels l'Etat de droit, l'unité nationale, la sécurité, la coopération étrangère et l'intégration africaine. Au plan économique et financier, le parti estime que le rôle de l'Etat doit essentiellement se baser sur la création et l'animation d'un cadre propice à l'éclosion et au développement des initiatives privées. Au plan socio-culturel, le parti mène une politique visant à l'amélioration des conditions de la femme, de l'éducation, de la santé, la protection de l'enfant, la promition de la jeunesse et la revalorisation du patrimoine culturel. ${ }^{73}$

\section{Conclusion}

Comme partout ailleurs en Afrique où des Constitutions ont vu le jour suite à des Conférences Nationales, il faut se demander, exemples du Congo et du Togo à l'appui, si le texte constitutionnel nigérien comporte en lui même assez de garanties pouvant permettre un passage en douceur du système ancien dans lequel la démocratie était inexistante à un système nouveau dans lequel la démocratie dans toute son ampleur sera appliquée.

La spécificité du Niger et le climat social qui y règne, avant le référendum constitutionnel et au lendemain de la promulgation de la Constitution, permet déjà d'augurer les nombreuses difficultés et résistances auxquelles seront confrontées les nouvelles institutions qui seront installées à l'issue des élections.

Ces difficultés ne doivent cependant pas occulter le fait que la nouvelle Constitution est un des premiers éléments traduisant l'évolution positive de l'histoire politique du Niger et la volonté de toutes les couches sociales de s'engager dans un processus démocratique devant aboutir à l'instauration d'un Etat de droit. L'application pratique de cette Constitution permettra d'évaluer son degré de performance, sa capacité à consacrer le libre exercice de la démocratie et à protéger les droits de la personne humaine.

73

Pour les trois derniers partis, la source est Le Républicain du 10 février 1992, p. 2 et Le Démocrate du lundi, 29 mars 1993, p. 3. 


\section{Freedom of Association, Ethnicity, and the Transition to Democracy}

\section{By Daniel Haile}

The introduction of new democratic governments in Africa is closely connected with the question of whether there can be democracy in Africa and how it should be safeguarded. Democracy is closely intertwined with development and with the overall situation of the society in which it is built up. The article gives an overview over the situation in Ethiopia regarding the development of a genuine respect for the ethnic, cultural and religious diversity of the country. It gives a closer look into how the right to form political parties and the recognition of collective rights are safeguarded in the 1991 Transitional Period Charter of Ethiopia.

After describing the Ethiopian situation, the article proceeds with a conceptual analysis of both legal concepts and comes to the conclusion that they are well balanced and soundly founded in the Ethiopian society and system.

The article, which is based on an address to the World Conference on Democracy in Washington in 1993, however, calls for patience in the evaluation of new concepts in a long process of a society, which has experienced unnecessary fratricidal wars and misery, in the evolution to a democratic system of government.

In a first overall evaluation of the Ethiopian transition process, the author considers the results to be encouraging.

\section{Democratic Elections in Niger}

\section{By Aichatou Dodo}

The article describes the constitutional and legislative framework of elections in the Niger at the levels of the Presidency of the Republic, the Deputies to the National Assembly, District and Municipal Councillors and of referenda. The distribution of seats in the National Assembly and other details of the electoral process are explained with reference to the elections which took place in 1993. Finally, the article sets out the legal status of the political parties as well as the principal objectives and electoral symbols of the parties taking part in the present elections. 